\title{
Echocardiography in arrhythmogenic right ventricular dysplasia/cardiomyopathy: Can the technology survive in the era of cardiac magnetic resonance imaging?
}

\author{
Rajesh Janardhanan \\ Department of Cardiovascular Medicine, Sarver Heart Center, \\ Banner-University Medical Center, Tucson, Arizona, United States
}

\section{Article p. 362}

Arrhythmogenic right ventricular dysplasia/ /cardiomyopathy (ARVD/C) is a genetically determined heart muscle disorder that is characterized pathologically by fibrofatty replacement of the right ventricular (RV) myocardium. The clinical diagnosis of $\mathrm{ARVD} / \mathrm{C}$ is complex, since there is no single diagnostic "gold standard". The diagnosis is based on major and minor criteria that have been modified and take into account heart structure and function, family history, genetic mutations, histology, ventricular arrhythmias, and electrocardiographic abnormalities [1].

Diagnostic imaging criteria include regional $\mathrm{RV}$ wall motion abnormalities in combination with RV dilatation or global RV systolic dysfunction [1]. Conventional echocardiography is capable of detecting these changes in the RV, consisting of visual regional wall motion analyses, outflow tract dimensions and RV-fractional area change, and remains part of the current diagnostic Task Force criteria [1].

However, a comprehensive and accurate evaluation of the RV by echocardiography is technically challenging due to the retrosternal position and complex geometry of the RV. Cardiac magnetic resonance (CMR) imaging has revolutionized the diagnostic accuracy for ARVD/C and found to be of superior value compared to conventional echocardiography [2]. Pathologies causing
RV volume overload such as intracardiac shunts (e.g. atrial septal defects, anomalous pulmonary venous drainage) can be misinterpreted as possible ARVD/C on standard echocardiography [3], and we often depend on CMR for a more definitive diagnosis.

Although the role of echocardiography versus $\mathrm{CMR}$ in $\mathrm{ARVD} / \mathrm{C}$ is subject to debate, it still remains the initial screening modality that can provide the first clue towards the diagnosis. In addition, CMR is often not feasible for the periodic follow-up of $\mathrm{ARVD} / \mathrm{C}$ due to the high rate of implantable cardioverter-defibrillator (ICD) implantation and the relative high cost. There have been recent advances in echocardiography with the availability of novel techniques such as 3 dimensional right ventricular (3D-RV) imaging and tissue deformation imaging. These methods could improve the diagnostic and prognostic performance of echocardiography in these patients.

In this issue of "Cardiology Journal", Mast et al. [4] describe the current and future role of both conventional and new echocardiographic parameters in ARVD/C with respect to diagnosis and follow-up. The authors provide an ARVD/C focused echocardiographic protocol which is currently in use in their institution. They discuss 3D-RV imaging which enables more accurate RV volumetric measurements compared to conventional echocardiography [5]. With the implementation of 3D-echocardiography, subtle global RV systolic

Address for correspondence: Rajesh Janardhanan, MD, MRCP, FACC, FASE, Associate Professor of Medicine and Medical Imaging, Medical Director, Non-Invasive Cardiac Imaging, Banner University Medical Center South, Sarver Heart Center, Box 245037, 1501 N. Campbell Avenue, Tucson, AZ 85724, USA, tel: 520 626 3766, fax: 520626 4333, e-mail: raj@shc.arizona.edu

Received: 04.06.2015 Accepted: 08.06.2000 
dysfunction can be seen in ARVD/C, and can contribute to early diagnosis [6].

The article also discusses the utility of tissue deformation imaging which can accurately detect subtle RV regional wall motion abnormalities and mechanical dyssynchrony [7, 8]. RV myocardial tissue deformation results primarily in longitudinal systolic shortening. The ability to quantify regional wall motion rather than visual qualitative assessment is a critical advantage of tissue deformation imaging compared to conventional echocardiographic wall motion analysis. The diagnostic performance of peak systolic strain has been found to be superior to conventional measurements in ARVD/C patients [9]. Both multiplane- and $3 \mathrm{D}$-speckle measurement of tracking are promising new applications in RV deformation imaging, but require further validation before widespread clinical implementation. Measurements of dyssynchrony could provide evidence of early, subtle electrical disturbances in ARVD/C, and may further improve the diagnostic value of echocardiography $[8,10]$.

The accurate diagnosis of ARVD/C is critically important because the diagnosis carries a risk of sudden cardiac death. An incorrect diagnosis can lead to unnecessary insertion of an ICD, cause a psychosocial burden, and initiate costly and extensive screening of family members, with the possibility of overdiagnosis due to inaccurate interpretation of tests performed during screening [11]. The article by Mast et al. [4] suggests that the novel robust parameters derived from 3D-RV echocardiography and tissue deformation imaging, has incremental diagnostic value and could change the current diagnostic role of echocardiography in $\mathrm{ARVD} / \mathrm{C}$.

Conflict of interest: None declared

\section{References}

1. Marcus FI, McKenna WJ, Sherrill D et al. Diagnosis of arrhythmogenic right ventricular cardiomyopathy/dysplasia: Proposed modification of the task force criteria. Circulation, 2010; 121: 1533-1541.

2. Borgquist R, Haugaa KH, Gilljam T et al. The diagnostic performance of imaging methods in arvc using the 2010 task force criteria. Eur Heart J Cardiovasc Imag, 2014; 15: 1219-1225.

3. Quarta G, Husain SI, Flett AS et al. Arrhythmogenic right ventricular cardiomyopathy mimics: Role of cardiovascular magnetic resonance. J Cardiovasc Magnetic Resonance, 2013; 15: 16.

4. Mast TP, Teske AJ, Doevendans PA, Cramer MJ. Current and future role of echocardiography in arrhythmogenic right ventricular dysplasia/cardiomyopathy. Cardiol J, 2015; 22: 362-374.

5. Prakasa KR, Dalal D, Wang J et al. Feasibility and variability of three dimensional echocardiography in arrhythmogenic right ventricular dysplasia/cardiomyopathy. Am J Cardiol, 2006; 97: 703-709.

6. Kjaergaard J. Assessment of right ventricular systolic function by tissue doppler echocardiography. Danish Med J, 2012; 59: B4409.

7. Teske AJ, De Boeck BW, Melman PG, Sieswerda GT, Doevendans PA, Cramer MJ. Echocardiographic quantification of myocardial function using tissue deformation imaging, a guide to image acquisition and analysis using tissue doppler and speckle tracking. Cardiovascular Ultrasound, 2007; 5: 27.

8. Sarvari SI, Haugaa KH, Anfinsen OG et al. Right ventricular mechanical dispersion is related to malignant arrhythmias: A study of patients with arrhythmogenic right ventricular cardiomyopathy and subclinical right ventricular dysfunction. Eur Heart J, 2011; 32: 1089-1096.

9. Teske AJ, Cox MG, De Boeck BW, Doevendans PA, Hauer RN, Cramer MJ. Echocardiographic tissue deformation imaging quantifies abnormal regional right ventricular function in arrhythmogenic right ventricular dysplasia/cardiomyopathy. J Am Soc Echocardiogr, 2009; 22: 920-927.

10. Tops LF, Prakasa K, Tandri H et al. Prevalence and pathophysiologic attributes of ventricular dyssynchrony in arrhythmogenic right ventricular dysplasia/cardiomyopathy. J Am Coll Cardiol, 2009; 54: 445-451.

11. Marcus F, Basso C, Gear K, Sorrell VL. Pitfalls in the diagnosis of arrhythmogenic right ventricular cardiomyopathy/dysplasia. Am J Cardiol, 2010; 105: 1036-1039. 